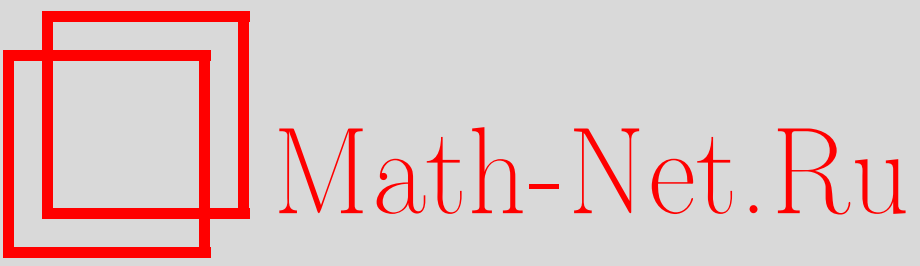

В. В. Панков, А. Д. Баев, В. Д. Харченко, А. А. Бабайцев, Априорная оценка решений одной краевой задачи в полосе для вырождающегося эллиптического уравнения высокого порядка, Итоги науки и техн. Сер. Соврем. мат. и ее прил. Темат. обз., 2019, том 173, 116-125

DOI: https://doi.org/10.36535/0233-6723-2019-173-116-125

Использование Общероссийского математического портала Math-Net.Ru подразумевает, что вы прочитали и согласны с пользовательским соглашением

http://www.mathnet.ru/rus/agreement

Параметры загрузки:

IP : 35.173 .219 .149

26 апреля 2023 г., 10:36:13 


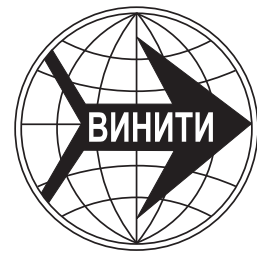

ИТОГИ НАУКИ И ТЕХНИКИ.

Современная математика и ее приложения.

Тематические обзоры.

Том 173 (2019). С. 116-125

DOI: $10.36535 / 0233-6723-2019-173-116-125$

УДК 517.956

\title{
АПРИОРНАЯ ОЦЕНКА РЕШЕНИЙ ОДНОЙ КРАЕВОЙ ЗАДАЧИ В ПОЛОСЕ ДЛЯ ВЫРОЖДАЮЩЕГОСЯ ЭЛЛИПТИЧЕСКОГО УРАВНЕНИЯ ВЫСОКОГО ПОРЯДКА
}

(c) 2019 г. В. В. ПАНКОВ, А. Д. БАЕВ, В. Д. ХАРЧЕНКО, А. А. БАБАЙЦЕВ

\begin{abstract}
АннотАция. Доказаны коэрцитивные априорные оценки решений краевой задачи типа задачи Дирихле в полосе для одного вырождающегося эллиптического уравнения высокого порядка, содержащего весовые производные специального вида до порядка $2 m$ и обычные частные производные до порядка $2 k-1$ при условии $2 m>2 k-1$. На границе полосы наложены условия типа Дирихле. Получена коэрцитивная априорная оценка решения рассматриваемой задачи. Оценка получена в специальных весовых пространствах типа пространств Соболева.
\end{abstract}

Ключевые слова: априорная оценка, вырождающееся эллиптическое уравнение, весовое пространство Соболева.

A PRIORI ESTIMATE OF SOLUTIONS OF ONE BOUNDARY-VALUE PROBLEM IN A STRIP FOR A HIGHER-ORDER DEGENERATE ELLIPTIC EQUATION

(c) 2019 V. V. PANKOV, A. D. BAEV, V. D. KHARCHENKO, A. A. BABAITSEV

\begin{abstract}
Coercive a priori estimates of solutions of a Dirichlet-type boundary-value problem in a strip for a certain higher-order degenerate elliptic equation containing weighted derivatives of a special form up to the order $2 m$ and ordinary partial derivatives up to the order $2 k-1$ under the condition $2 m>2 k-1$ are proved. At the boundary of the strip, Dirichlet-type conditions are imposed. A coercive a priori estimate for solutions of the problem considered in special weighted Sobolev-type spaces is obtained.
\end{abstract}

Keywords and phrases: a priori estimate, degenerate elliptic equation, Sobolev weight space.

AMS Subject Classification: 35S05, 35S11

Введение. Теория краевых задач для вырождающихся эллиптических уравнений в настоящее время интенсивно развивается. Краевые задачи для таких уравнений относятся к «неклассическим» задачам математической физики. Одна из главных трудностей, возникающих в теории вырождающихся эллиптических уравнений, связана с влиянием младших (в смысле теории регулярных эллиптических операторов) членов уравнения на постановку граничных задач и их коэрцитивную разрешимость.

Работа выполнена при поддержке Министерства образования и науки Российской Федерации (проект № 14.Z50.31.0037). 
Исследование вырождающихся эллиптических уравнений высокого порядка (при «степенном» характере вырождения) было начато в работах М. И. Вишика и В. В. Грушина (см. $[7,8])$. В работе В. П. Глушко [9] были доказаны априорные оценки краевых задач для уравнений, вырождающихся на границе в уравнение первого порядка по одной из переменных. В работах А. Д. Баева [1-3] были получены априорные оценки и теоремы о существовании решений краевых задач для вырождающихся эллиптических уравнений высокого порядка при произвольном сильном характере вырождения. В частности, были исследованы краевые задачи в полосе для уравнений высокого порядка, вырождающихся на границе области в уравнение четного порядка. В работах А. Д. Баева и С. С. Бунеева $[4,5]$ были исследованы краевые задачи в полосе для эллиптических уравнений высокого порядка, вырождающихся на границе в уравнение третьего порядка. В [10] были получены коэрцитивные априорные оценки для одного эллиптического уравнения высокого порядка, вырождающегося на границе $t=0$ в уравнение нечетного порядка.

В настоящей работе получены априорные оценки решений краевых задач в полосе для уравнений высокого порядка, вырождающихся на границе в уравнение нечетного порядка по одной из переменных. Таким образом, работа является естественным продолжением исследований, начатых в $[4,5,10]$. Формулировка полученных результатов содержится в [6].

1. Основные определения и результаты. В полосе $\mathbb{R}_{d}^{n}=\left\{x \in \mathbb{R}^{n-1}, 0<t<d\right\}$, где $d>0-$ некоторое число, рассмотрим уравнение

$$
A\left(D_{x}, D_{\alpha, t}, \partial_{t}\right) v(x, t)=F(x, t)
$$

где

$$
A\left(D_{x}, D_{\alpha, t}, \partial_{t}\right) v=L_{2 m}\left(D_{x}, D_{\alpha, t}\right) v-b(-1)^{k} \partial_{t}^{2 k-1} v, \quad L_{2 m}\left(D_{x}, D_{\alpha, t}\right)=\sum_{|\tau|+j \leqslant 2 m} a_{\tau j} D_{x}^{\tau} D_{\alpha, t}^{j},
$$

$a_{\tau j}$-комплексные числа, $\operatorname{Im} \bar{b} a_{0,2 m}=0$. Здесь

$$
D_{\alpha, t}=\frac{1}{i} \sqrt{\alpha(t)} \partial_{t} \sqrt{\alpha(t)}, \quad \partial_{t}=\frac{\partial}{\partial t}, \quad D_{x}^{\tau}=i^{|\tau|} \partial_{x_{1}}^{\tau_{1}} \partial_{x_{2}}^{\tau_{2}} \ldots \partial_{x_{n-1}}^{\tau_{n-1}}
$$

На границе $t=0$ полосы $\mathbb{R}_{d}^{n}$ задаются условия

$$
\left.B_{j}\left(D_{x}\right) v\right|_{t=0}=\left.\sum_{|\tau| \leqslant m_{j}} b_{\tau j} D_{x}^{\tau} \partial_{t}^{j-1} v\right|_{t=0}=G_{j}(x), \quad j=1,2, \ldots, k,
$$

с комплексными коэффициентами $b_{\tau j}$. На границе $t=d$ полосы $\mathbb{R}_{d}^{n}$ заданы условия вида

$$
\left.v\right|_{t=d}=\left.\partial_{t} v\right|_{t=d}=\cdots=\left.\partial_{t}^{m-1} v\right|_{t=d}=0
$$

Уравнение вида

где

$$
A\left(D_{x}, D_{\alpha, t}, \partial_{t}\right) v(x, t)=F(x, t)
$$

$$
A\left(D_{x}, D_{\alpha, t}, \partial_{t}\right) v=L_{2 m}\left(D_{x}, D_{\alpha, t}\right) v+b(-1)^{k} \partial_{t}^{2 k-1} v,
$$

было исследовано в [10]; п ри этом на границе $t=0$ надо было ставить на одно условие меньше.

Предположим, что выполнены следующие условия.

Условие 1. При всех $(\xi, \eta) \in \mathbb{R}^{n}$ справедливо неравенство

$$
\operatorname{Re} \bar{b} L_{2 m}(\xi, \eta) \geqslant c\left(1+|\xi|^{2}+|\eta|^{2}\right)^{m}
$$

где постоянная $c>0$ не зависит от $(\xi, \eta)$.

Условие 2. Для некоторого $s \geqslant 2 m+\max _{1 \leqslant j \leqslant k-1}\left(m_{j}\right)$ функция $\alpha(t)$ принадлежит $C^{s-1}[0, d]$, причем $\alpha(0)=\alpha^{\prime}(0)=0, \alpha(t)>0$ при $t>0$.

Условие 3. При всех $\xi \in \mathbb{R}^{n-1}$ выполняется неравенство

$$
\sum_{|\tau| \leqslant m_{j}} b_{\tau j} \xi^{\tau} \neq 0, \quad j=1,2, \ldots, k
$$


Определим пространства, в которых будет исследоваться задача (1)-(3). Введем в рассмотрение интегральное преобразование, которое на функциях $u(t) \in C_{0}^{\infty}\left(\mathbb{R}_{+}^{1}\right)$ записывается в виде

$$
F_{\alpha}[u(t)](\eta)=\int_{0}^{+\infty} u(t) \exp \left(i \eta \int_{t}^{d} \frac{d \rho}{\alpha(\rho)}\right) \frac{d t}{\sqrt{\alpha(t)}} .
$$

Это преобразование было введено в [1]. Там же было отмечено, что для преобразования $F_{\alpha}$ можно построить обратное преобразование:

$$
F_{\alpha}^{-1}[w(\eta)](t)=\left.\frac{1}{\sqrt{\alpha(t)}} F_{\eta \rightarrow \tau}^{-1}[w(\eta)]\right|_{\tau=\phi(t)},
$$

где $F_{\eta \rightarrow \tau}^{-1}$ - обратное преобразование Фурье. В [1] было доказано, что для преобразования $F_{\alpha}$ справедливо равенство, являющееся аналогом равенства Парсеваля. Это дает возможность рассмотреть его не только на функциях из $L_{2}\left(\mathbb{R}_{+}^{1}\right)$, но и на некоторых классах обобщенных функций.

Определение 1. Пространство $H_{s, \alpha, \frac{2 m}{2 k-1}}\left(\mathbb{R}_{d}^{n}\right)(s \geqslant 0$ - целое число) состоит из тех функций $v(x, t) \in L_{2}\left(\mathbb{R}_{d}^{n}\right)$, для которых конечна норма

$$
\|v\|_{s, \alpha, m}=\left\{\sum_{l=0}^{\left[\frac{(2 k-1) s}{2 m}\right]}\left\|F_{\xi \rightarrow x}^{-1} F_{\alpha}^{-1}\left[\left(1+|\xi|^{2}+|\eta|^{2}\right)^{\frac{1}{2}\left(s-\frac{2 m}{2 k-1} l\right)} F_{\alpha} F_{x \rightarrow \xi}\left[\partial_{t}^{l} v(x, t)\right]\right]\right\|_{L_{2}\left(\mathbb{R}_{d}^{n}\right)}^{2}\right\}^{1 / 2},
$$

где [·] означает целую часть числа.

Если $s$ - такое натуральное число, что $(2 k-1) s /(2 m)$ - целое число, то эта норма эквивалентна следующей норме:

$$
\|v\|_{s, \alpha, q}=\left\{\sum_{|\tau|+j+\frac{2 m}{2 k-1} l \leqslant s}\left\|D_{x}^{\tau} D_{\alpha, t}^{j} \partial_{t}^{l} v\right\|_{L_{2}\left(\mathbb{R}_{d}^{n}\right)}^{2}\right\}^{1 / 2} .
$$

Основным результатом работы является следующее утверждение.

Теорема 1. Предположим, что

$$
s \geqslant \max \left\{2 m, \max _{1 \leqslant j \leqslant k}\left(m_{j}+\frac{2 m(j-1)}{2 k-1}\right)+\frac{m}{2 k-1}\right\}
$$

- целое число, $m \geqslant 2 k-1$ - целое число, и выполнены условия 1-3. Тогда для любого решения $v(x, t)$ задачи (1)-(3), принадлежащего пространству $H_{s, \alpha, 2 m / 3}\left(\mathbb{R}_{d}^{n}\right)$, справедлива априорная оценка

$$
\|v\|_{s, \alpha, \frac{2 m}{2 k-1}} \leqslant c\left(\|A v\|_{s-2 m, \alpha, \frac{2 m}{2 k-1}}+\sum_{j=1}^{k}\left\|\left.B_{j} v\right|_{t=0}\right\|_{s-m_{j}-\frac{2 m(j-1)}{2 k-1}-\frac{m}{2 k-1}}\right),
$$

где постоянная $c>0$ не зависит от $v$.

Здесь $\|\cdot\|_{s}$ - норма в пространстве Соболева-Слободецкого $H_{s}\left(\mathbb{R}^{n-1}\right)$.

Формулировка теоремы 1 содержится в [6].

2. Схема доказательства теоремы 1. Если применить к обеим частям уравнения (1) и условий (2)-(3) преобразование Фурье $F_{x \rightarrow \xi}$, то получим следующую задачу, зависящую от параметра $\xi \in \mathbb{R}^{n-1}$ :

$$
\begin{gathered}
A\left(\xi, D_{\alpha, t}, \partial_{t}\right) u(\xi, t)=L_{2 m}\left(\xi, D_{\alpha, t}\right) u(\xi, t)-b(-1)^{k} \partial_{t}^{2 k-1} u(\xi, t)=f(\xi, t), \\
\left.B_{j}(\xi) u\right|_{t=0}=\left.\sum_{|\tau| \leqslant m_{j}} b_{\tau j} \xi^{\tau} \partial_{t}^{j-1} u\right|_{t=0}=g_{j}(\xi), \quad j=1,2, \ldots, k, \\
\left.u(\xi, t)\right|_{t=d}=\left.\partial_{t} u(\xi, t)\right|_{t=d}=\cdots=\left.\partial_{t}^{m-1} u(\xi, t)\right|_{t=d}=0 .
\end{gathered}
$$


Здесь

$$
u(\xi, t)=F_{x \rightarrow \xi}[\nu(x, t)], \quad f(\xi, t)=F_{x \rightarrow \xi}[F(x, t)], \quad g(\xi)=F_{x \rightarrow \xi}[G(x)] .
$$

Аналогично определенным выше пространствам введем пространства $\widetilde{H}_{s, \alpha, \frac{2 m}{2 k-1}}(0 ; d)$.

Определение 2. Будем говорить, что функция $u(t)$ принадлежит пространству $\widetilde{H}_{s, \alpha, \frac{2 m}{2 k-1}}(0 ; d)$ $\left(s \geqslant 0\right.$ - целое число), если конечна следующая норма, зависящая от параметра $\xi \in \mathbb{R}^{n-1}$ :

$$
\|u\|_{s, \alpha, \frac{2 m}{2 k-1},|\xi|}=\left\{\sum_{k+\frac{2 m}{2 k-1} j \leqslant s}\left\|F_{\alpha}^{-1}\left[\left(1+|\xi|^{2}+\eta^{2}\right)^{k / 2} F_{\alpha}\left[\partial_{t}^{j} u\right]\right]\right\|_{L_{2}(0 ; d)}^{2}\right\}^{1 / 2} .
$$

Теорема 1 выводится из следующего утверждения.

Теорема 2. Предположим, что

$$
s \geqslant \max \left\{2 m, \max _{1 \leqslant j \leqslant k}\left(m_{j}+\frac{2 m(j-1)}{2 k-1}\right)+\frac{m}{2 k-1}\right\},
$$

$m \geqslant 2 k-1$, причем $s, m-$ целые числа. Пусть $f(\xi, t) \in \widetilde{H}_{s-2 m, \alpha, \frac{2 m}{2 k-1}}(0 ; d)$ nри всех $\xi \in \mathbb{R}^{n-1} u$ выполнены условия 1-3. Тогда для любого решения и $(\xi, t)$ задачи (4)-(6), принадлежащего при всех $\xi \in \mathbb{R}^{n-1}$ пространству $\widetilde{H}_{s, \alpha, \frac{2 m}{2 k-1}}(0 ; d)$, справедлива априорная оченка

$$
\|u\|_{s, \alpha, \frac{2 m}{2 k-1},|\xi|}^{2} \leqslant c\left(\|f\|_{s-2 m, \alpha, \frac{2 m}{2 k-1},|\xi|}^{2}+\sum_{j=1}^{k}\left(1+|\xi|^{2}\right)^{s-m_{j}-\frac{2 m(j-1)}{2 k-1}-\frac{m}{2 k-1}}\left|g_{j}(\xi)\right|^{2}\right)
$$

с константой с $>0$, не зависящей от $\xi \in \mathbb{R}^{n-1}, u, f, g$.

Из определения преобразования $F_{\alpha}$ получим, что для любых $u(t) \in L_{2}(0 ; d), w(t) \in L_{2}(0 ; d)$ справедливо равенство

$$
\int_{-\infty}^{\infty} F_{\alpha}[u](\eta) \cdot \overline{F_{\alpha}[w](\eta)} d \eta=2 \pi(u, w)
$$

здесь и в дальнейшем через $(\cdot, \cdot)$ обозначается скалярное произведение в $L_{2}(0 ; d)$.

Кроме того, из определения преобразования $F_{\alpha}$ следует, что если $u(t) \in C^{s}[0 ; d]$ удовлетворяет условиям

то справедливо равенство

$$
u(d)=\partial_{t} u(d)=\cdots=\partial_{t}^{s-1}(d)=0,
$$

$$
F_{\alpha}\left[D_{\alpha, t}^{j} u\right](\eta)=\eta^{j} F_{\alpha}[u](\eta) \quad \forall j=0,1,2, \ldots, s .
$$

Из (10) следует, что если $u(t) \in C^{s}[0 ; d], w(t) \in C^{s}[0 ; d]$ и эти функции удовлетворяют условиям (9), то справедливо равенство

$$
\left(D_{\alpha, t}^{j} u(t), w(t)\right)=\frac{1}{2 \pi} \int_{-\infty}^{\infty} \eta^{j} F_{\alpha}[u](\eta) \overline{F_{\alpha}[w](\eta)} d \eta .
$$

В дальнейшем нам понадобятся аналоги неравенства Эрлинга-Ниренберга для весовых производных, которые в нашем случае можно сформулировать следующим образом.

Лемма 1. Если $u(t) \in \tilde{H}_{s, \alpha, \frac{2 m}{2 k-1}}(0 ; d)(s-$ натуральное число), то при любых $\varepsilon>0 u j=$ $0,1,2, \ldots, s-1$ справедливо неравенство

с константой $c>0$, не зависящей от и.

$$
\left\|D_{\alpha, t}^{j} u\right\|^{2} \leqslant \varepsilon^{2(s-j)}\left\|D_{\alpha, t}^{s} u\right\|^{2}+\left(c \varepsilon^{-2 j}+\varepsilon^{2(s-j)}\right)\|u\|^{2}
$$

Здесь и в дальнейшем через $\|\cdot\|$ обозначается норма в пространстве $L_{2}(0 ; d)$. 
Следствие 1. Если $u(t) \in \tilde{H}_{s, \alpha, \frac{2 m}{2 k-1}}(0 ; d)$, то для любых $\varepsilon>0, j=0,1,2, \ldots, 2 m-1, \xi \in \mathbb{R}^{n-1}$ справедливо неравенство

$$
\left(1+|\xi|^{2}\right)^{2 m-j}\left\|D_{\alpha, t}^{j} u\right\|^{2} \leqslant \varepsilon^{2(2 m-j)}\left\|D_{\alpha, t}^{2 m} u\right\|^{2}+c(\varepsilon)\left(1+|\xi|^{2}\right)^{2 m}\|u\|^{2}
$$

с константой с $>0$, не зависящей от $u, \xi$.

Теорема 2 получается из доказанных ниже лемм, где константы $c>0, \varepsilon>0$ во всех оценках не зависят от $u, \xi$.

Лемма 2. Пусть выполнены условия 1 и 2 , а такэе $m \geqslant 2 k-1$. Тогда для любой функиии $u(t) \in \tilde{H}_{2 m, \alpha, \frac{2 m}{2 k-1}}(0 ; d)$ справедлива оченка

$$
\begin{aligned}
\sum_{j=0}^{m}\left(1+|\xi|^{2}\right)^{2 m-j}\left\|D_{\alpha, t}^{j} u\right\|^{2} & \leqslant c\left(\left\|A\left(\xi, D_{\alpha, t}, \partial_{t}\right) u(t)\right\|^{2}+\right. \\
& \left.+\left(1+|\xi|^{2}\right)^{m} \sum_{j=0}^{k-2}(-1)^{k-j-1} \operatorname{Re} \partial_{t}^{2 k-2-j} u(0) \cdot \overline{\partial_{t}^{j} u(0)}+\left|\partial_{t}^{k-1} u(0)\right|^{2}\right)
\end{aligned}
$$

с константой с $>0$, не зависящей от $\xi, u$.

Доказательство. Так как пространство $C^{2 m}[0 ; d]$ плотно в пространстве $\tilde{H}_{2 m, \alpha, \frac{2 m}{2 k-1}}(0 ; d)$, то неравенство (14) достаточно доказать для функций из пространства $C^{2 m}[0 ; d]$. Умножив скалярно в $L_{2}(0, d)$ обе части уравнения (4) на функцию $b u(t)$, получим

$$
\operatorname{Re}\left(L_{2 m}\left(\xi, D_{\alpha, t}\right) u, b u\right)-|b|^{2}(-1)^{k} \operatorname{Re}\left(\partial_{t}^{2 k-1} u, u\right)=\operatorname{Re}\left(A\left(\xi, D_{\alpha, t}, \partial_{t}\right) u, b u\right) .
$$

Используя равенство (10) и условие 1 , получим оценку

$$
\operatorname{Re}\left(L_{2 m}\left(\xi, D_{\alpha, t}\right) u, b u\right) \geqslant c_{1} \sum_{j=0}^{m}\left(1+|\xi|^{2}\right)^{m-j}\left\|D_{\alpha, t}^{j} u\right\|,
$$

где $c_{1}>0$ - некоторая константа, не зависящая от $\xi \in \mathbb{R}^{n-1}, u$.

С использованием условия (3) получим равенство

$$
(-1)^{k} \int_{0}^{d} \partial_{t}^{2 k-1} u \cdot \bar{u} d t=\sum_{j=0}^{k-2}(-1)^{k-j-1} \partial_{t}^{2 k-2-j} u(0) \cdot \overline{\partial_{t}^{j} u(0)}+\frac{1}{2}\left|\partial_{t}^{k-1} u(0)\right|^{2} .
$$

Применяя (16), (17) в (15) и используя неравенство Коши-Буняковского, получим для любого $\varepsilon>0$ оценку

$$
\begin{aligned}
& c_{1} \sum_{j=0}^{m}\left(1+|\xi|^{2}\right)^{2 m-j}\left\|D_{\alpha, t}^{j} u\right\|^{2}-\frac{1}{2}|b|^{2}\left|\partial_{t}^{k-1} u(0)\right|^{2}\left(1+|\xi|^{2}\right)^{m} \leqslant \\
& \leqslant \frac{1}{\varepsilon}\left\|A\left(\xi, D_{\alpha, t}, \partial_{t}\right) u(t)\right\|^{2}+\varepsilon\left(1+|\xi|^{2}\right)^{2 m}\|u\|^{2}+\left(1+|\xi|^{2}\right)^{m}|b|^{2} \sum_{j=0}^{k-2}(-1)^{k-j-2} \partial_{t}^{2 k-2-j} u(0) \cdot \overline{\partial_{t}^{j} u(0)} .
\end{aligned}
$$

Выбирая в этом неравенстве $\varepsilon>0$ достаточно малым, получим оценку (14).

Лемма 3. При выполнении условий леммы 2 для любой функции $и(t) \in \tilde{H}_{2 m, \alpha, \frac{2 m}{2 k-1}}(0 ; d)$ справедлива оченка

$$
\left\|D_{\alpha, t}^{2 m} u\right\|^{2} \leqslant \varepsilon\left(\left\|D_{\alpha, t}^{2 m} u\right\|^{2}+\left\|\partial_{t}^{2 k-1} u\right\|^{2}\right)+c(\varepsilon)\left(\left\|A\left(\xi, D_{\alpha, t}, \partial_{t}\right) u\right\|^{2}+\left(1+|\xi|^{2}\right)^{2 m}\|u\|^{2}\right)
$$

при любом $\varepsilon>0$. 
Доказательство. Умножив скалярно обе части равенства (4) на $a_{0,2 m} D_{\alpha, t}^{2 m} u$, получим оценку

$$
\begin{aligned}
\left|a_{0,2 m}\right|^{2 m}\left\|D_{\alpha, t}^{2 m} u\right\|^{2}-\operatorname{Re}\left(b(-1)^{k} \partial_{t}^{2 k-1} u, a_{0,2 m} D_{\alpha, t}^{2 m} u\right) \leqslant & \\
& \leqslant\left|\sum_{\substack{|\tau|+j \leqslant 2 m \\
|\tau| \geqslant 1}} a_{\tau j} \xi^{\tau}\left(D_{\alpha, t}^{j} u, a_{0,2 m} D_{\alpha, t}^{2 m} u\right)\right|+\left|\left(A\left(\xi, D_{\alpha, t}, \partial_{t}\right) u(t), a_{0,2 m} D_{\alpha, t}^{2 m} u\right)\right| \cdot
\end{aligned}
$$

Заметим, что

$$
\left|\left(A\left(\xi, D_{\alpha, t}, \partial_{t}\right) u(t), a_{0,2 m} D_{\alpha, t}^{2 m} u\right)\right| \leqslant \varepsilon\left\|D_{\alpha, t}^{2 m} u\right\|^{2}+\frac{1}{\varepsilon}\left\|A\left(\xi, D_{\alpha, t}, \partial_{t}\right) u(t)\right\|^{2} .
$$

С помощью (13) и неравенства Коши-Буняковского получим для любого $\varepsilon>0$ оценку

$$
\left|\sum_{\substack{|\tau|+j \leqslant 2 m \\|\tau| \geqslant 1}} a_{\tau j} \xi^{\tau} a_{0,2 m}\left(D_{\alpha, t}^{j} u, D_{\alpha, t}^{2 m} u\right)\right| \leqslant \varepsilon\left\|D_{\alpha, t}^{2 m} u\right\|^{2}+c_{1}(\varepsilon)\left(1+|\xi|^{2}\right)^{2 m}\|u\|^{2} .
$$

Используя (20) и (21) в правой части (19), получим оценку

$$
\begin{aligned}
& \left|a_{0,2 m}\right|^{2 m}\left\|D_{\alpha, t}^{2 m} u\right\|^{2}-\operatorname{Re}\left(b(-1)^{k} \partial_{t}^{2 k-1} u, a_{0,2 m} D_{\alpha, t}^{2 m} u\right) \leqslant \\
& \quad \leqslant \varepsilon\left\|D_{\alpha, t}^{2 m} u\right\|^{2}+c_{2}(\varepsilon)\left(\left\|A\left(\xi, D_{\alpha, t}, \partial_{t}\right) u\right\|^{2}+\left(1+|\xi|^{2}\right)^{2 m}\|u\|^{2}\right) .
\end{aligned}
$$

Заметим, что

$$
\operatorname{Re}\left(\partial_{t}^{2 k-1} u, D_{\alpha, t}^{2 m} u\right)=(-1)^{k-1} \operatorname{Re}\left(\partial_{t}^{k} u, \partial_{t} D_{\alpha, t}^{2 m} \partial_{t}^{k-2} u\right)+\sum_{j=1}^{k-2}(-1)^{j} \operatorname{Re} K_{j},
$$

где операторы $K_{j}$ определены формулой

$$
K_{j}=I_{2 m, 1}\left(D_{\alpha, t}, \partial_{t}\right) \partial_{t}^{j-1} u
$$

Здесь $I_{2 m, 1}\left(D_{\alpha, t}, \partial_{t}\right)$ - коммутатор операторов $D_{\alpha, t}^{2 m}$ и $\partial_{t}$. Интегрируя по частям, получим равенство

$$
\begin{aligned}
\operatorname{Re}\left(\partial_{t}^{2 k-1} u, D_{\alpha, t}^{2 m} u\right)=(-1)^{k}\left(D_{\alpha, t}^{m} \partial_{t}^{k} u, D_{\alpha, t}^{m} \partial_{t}^{k-1} u\right) & +\sum_{j=1}^{k-1}(-1)^{j} K_{j}+ \\
& +\left.(-1)^{k} \sum_{j=0}^{m-1} i \alpha(t) D_{\alpha, t}^{j} \partial_{t}^{k} u \cdot D_{\alpha, t}^{2 m-j-1} \partial_{t}^{k-1} \bar{u}\right|_{t=d}
\end{aligned}
$$

Так как при $j \leqslant m-1-k$ выполняется неравенство $j+k \leqslant m-1$, то, учитывая граничные условия (6), имеем

$$
\left.\sum_{j=0}^{m-1} i \alpha(t) D_{\alpha, t}^{j} \partial_{t}^{k} u \cdot D_{\alpha, t}^{2 m-j-1} \partial_{t}^{k-1} \bar{u}\right|_{t=d}=\left.\sum_{j=m-k}^{m-1} i \alpha(t) D_{\alpha, t}^{j} \partial_{t}^{k} u \cdot D_{\alpha, t}^{2 m-j-1} \partial_{t}^{k-1} \bar{u}\right|_{t=d}
$$


Таким образом, получаем

$$
\begin{gathered}
\operatorname{Re}\left(\partial_{t}^{2 k-1} u, D_{\alpha, t}^{2 m} u\right)=(-1)^{k} \operatorname{Re}\left(D_{\alpha, t}^{m} \partial_{t}^{k} u, D_{\alpha, t}^{m} \partial_{t}^{k-1} u\right)+\sum_{j=1}^{k-1}(-1)^{j} \operatorname{Re} K_{j}+ \\
+\left.(-1)^{k} \operatorname{Re} \sum_{j=m-k}^{m-1} i \alpha(t) D_{\alpha, t}^{j} \partial_{t}^{k} u \cdot D_{\alpha, t}^{2 m-j-1} \partial_{t}^{k-1} \bar{u}\right|_{t=d}=(-1)^{k} \operatorname{Re}\left(\partial_{t} D_{\alpha, t}^{m} \partial_{t}^{k-1} u, D_{\alpha, t}^{m} \partial_{t}^{k-1} u\right)+ \\
+\sum_{j=1}^{k-1}(-1)^{j} \operatorname{Re} K_{j}+\left.(-1)^{k} \operatorname{Re} \sum_{j=m-k}^{m-1} i \alpha(t) D_{\alpha, t}^{j} \partial_{t}^{k} u \cdot D_{\alpha, t}^{2 m-j-1} \partial_{t}^{k-1} \bar{u}\right|_{t=d}+ \\
+(-1)^{k} \operatorname{Re}\left(I_{m, 1}\left(D_{\alpha, t}^{m}, \partial_{t}\right) \partial_{t}^{k-1} u, D_{\alpha, t}^{m} \partial_{t}^{k-1} u\right) .
\end{gathered}
$$

Здесь $I_{m, 1}\left(D_{\alpha, t}^{m}, \partial_{t}\right)$ - коммутатор операторов $D_{\alpha, t}^{m}$ и $\partial_{t}$. Так как

$$
\operatorname{Re}\left(\partial_{t} D_{\alpha, t}^{m} \partial_{t}^{k-1} u, D_{\alpha, t}^{m} \partial_{t}^{k-1} u\right)=0
$$

то справедливо равенство

$$
\begin{aligned}
\operatorname{Re}\left(\partial_{t}^{2 k-1} u, D_{\alpha, t}^{2 m} u\right)= & \sum_{j=1}^{k-1}(-1)^{j} \operatorname{Re} K_{j}+\left.(-1)^{k} \operatorname{Re} \sum_{j=m-k}^{m-1} i \alpha(t) D_{\alpha, t}^{j} \partial_{t}^{k} u \cdot D_{\alpha, t}^{2 m-j-1} \partial_{t}^{k-1} \bar{u}\right|_{t=d}+ \\
& +(-1)^{k} \operatorname{Re}\left(I_{m, 1}\left(D_{\alpha, t}^{m}, \partial_{t}\right) \partial_{t}^{k-1} u, D_{\alpha, t}^{m} \partial_{t}^{k-1} u\right) .
\end{aligned}
$$

Используя это равенство в (22), получим оценку

$$
\begin{aligned}
&\left|a_{0,2 m}\right|^{2 m}\left\|D_{\alpha, t}^{2 m} u\right\|^{2} \leqslant \varepsilon\left\|D_{\alpha, t}^{2 m} u\right\|^{2}+c_{2}(\varepsilon)\left(\left\|A\left(\xi, D_{\alpha, t}, \partial_{t}\right) u\right\|^{2}+\left(1+|\xi|^{2}\right)^{2 m}\|u\|^{2}\right)+ \\
&+\left(\sum_{j=1}^{k-1}\left|\operatorname{Re} K_{j}\right|+\left|\operatorname{Re} \sum_{j=m-k}^{m-1} i \alpha(t) D_{\alpha, t}^{j} \partial_{t}^{k} u \cdot D_{\alpha, t}^{2 m-j-1} \partial_{t}^{k-1} \bar{u}\right|_{t=d} \mid+\right. \\
&\left.+\left|\operatorname{Re}\left(I_{m, 1}\left(D_{\alpha, t}^{m}, \partial_{t}\right) \partial_{t}^{k-1} u, D_{\alpha, t}^{m} \partial_{t}^{k-1} u\right)\right|\right) .
\end{aligned}
$$

С помощью неравенства Коши-Буняковского и (13) получим

$$
\begin{aligned}
\sum_{j=1}^{k-1}\left|\operatorname{Re} K_{j}\right|+\mid \operatorname{Re}\left(I_{m, 1}\left(D_{\alpha, t}^{m}, \partial_{t}\right) \partial_{t}^{k-1} u\right. & \left., D_{\alpha, t}^{m} \partial_{t}^{k-1} u\right) \mid \leqslant \\
& \leqslant \varepsilon\left(\left\|\partial_{t}^{2 k-1} u\right\|^{2}+\left\|D_{\alpha, t}^{2 m} u\right\|^{2}\right)+c_{3}(\varepsilon)\left(1+|\varepsilon|^{2}\right)^{2 m}\|u\|^{2}
\end{aligned}
$$

Учитывая известную теорему «о следах», имеем

$$
\begin{aligned}
& \left.\left|\operatorname{Re} \sum_{j=m-k}^{m-1} i \alpha(t) D_{\alpha, t}^{j} \partial_{t}^{k} u \cdot D_{\alpha, t}^{2 m-j-1} \partial_{t}^{k-1} \bar{u}\right|_{t=d}\left|\leqslant c \sum_{j=1}^{2 m-1}\right| \partial_{t}^{j} u(d)\right|^{2} \leqslant \\
& \leqslant \varepsilon_{1} \int_{d / 2}^{d}\left|\partial_{t}^{2 m} u\right|^{2} d t+c_{4}\left(\varepsilon_{1}\right) \int_{d / 2}^{d}|u(t)|^{2} d t \leqslant \varepsilon\left\|D_{\alpha, t}^{2 m} u\right\|^{2}+c_{5}(\varepsilon) \cdot\left(1+|\xi|^{2}\right)^{2 m}\|u\|^{2} .
\end{aligned}
$$

Используя (25), (26) в правой части неравенства (23), получим, выбирая $\varepsilon>0$ достаточно малым, оценку (18). 
Лемма 4. При выполнении условий леммы 2 для любой функции и $(t) \in \tilde{H}_{2 m, \alpha, \frac{2 m}{2 k-1}}(0 ; d)$ справедлива оченка

$$
\left\|\partial_{t}^{2 k-1} u\right\|^{2} \leqslant c\left(\left\|A\left(\xi, D_{\alpha, t}, \partial_{t}\right) u\right\|^{2}+\left(1+|\xi|^{2}\right)^{2 m}\|u\|^{2}\right)
$$

Доказательство. Из уравнения (4) получим с помощью неравенства (13):

$$
\left\|\partial_{t}^{2 k-1} u\right\|^{2} \leqslant\left\|A\left(\xi, D_{\alpha, t}, \partial_{t}\right) u\right\|^{2}+\left\|D_{\alpha, t}^{2 m} u\right\|^{2}+\varepsilon\left\|D_{\alpha, t}^{2 m} u\right\|^{2}+c(\varepsilon)\left(1+|\xi|^{2}\right)^{2 m}\|u\|^{2}
$$

при любом $\varepsilon>0$. Применяя (18) и выбирая $\varepsilon>0$ достаточно малым, получим оценку (27).

Доказательство теоремы 2. Используя леммы 2-4, получим

$$
\begin{aligned}
\|u\|_{s, \alpha, \frac{2 m}{2 k-1},|\xi|}^{2} \leqslant & c\left(\|f\|_{s-2 m, \alpha, \frac{2 m}{2 k-1},|\xi|}^{2}+\right. \\
& \left.+\left(1+|\xi|^{2}\right)^{m} \sum_{j=0}^{k-2}(-1)^{k-j} \operatorname{Re} \partial_{t}^{2 k-2-j} u(0) \cdot \overline{\partial_{t}^{j} u(0)}+\left(1+|\xi|^{2}\right)^{m}\left|\partial_{t}^{k-1} u(0)\right|^{2}\right) .
\end{aligned}
$$

Используя неравенство Коши, получим оценку

$$
\begin{aligned}
\left|\left(1+|\xi|^{2}\right)^{m} \sum_{j=0}^{k-2}(-1)^{k-j} \operatorname{Re} \partial_{t}^{2 k-2-j} u(0) \cdot \overline{\partial_{t}^{j} u(0)}\right| & \leqslant \\
& \leqslant\left(1+|\xi|^{2}\right)^{m} \sum_{j=0}^{k-2}\left(\varepsilon\left|\partial_{t}^{2 k-2-j} u(\xi, 0)\right|^{2}+\frac{1}{\varepsilon}\left|\partial_{t}^{j} u(\xi, 0)\right|^{2}\right)
\end{aligned}
$$

для любого $\varepsilon>0$. Выбирая в этом неравенстве $\varepsilon=\varepsilon_{1}\left(1+|\xi|^{2}\right)^{-m+q j+q / 2}$, где $q=2 m /(2 k-1)$, имеем

$$
\begin{aligned}
& \left|\left(1+|\xi|^{2}\right)^{m} \sum_{j=0}^{k-2}(-1)^{k-j} \operatorname{Re} \partial_{t}^{2 k-2-j} u(0) \cdot \overline{\partial_{t}^{j} u(0)}\right| \leqslant \\
& \quad \leqslant \sum_{j=0}^{k-2}\left(\varepsilon_{1}\left(1+|\xi|^{2}\right)^{q j+q / 2}\left|\partial_{t}^{2 k-2-j} u(\xi, 0)\right|^{2}+\frac{1}{\varepsilon_{1}}\left(1+|\xi|^{2}\right)^{2 m-q j-q / 2}\left|\partial_{t}^{j} u(\xi, 0)\right|^{2}\right),
\end{aligned}
$$

где $\varepsilon_{1}-$ любое число. Заметим, что

$$
\left|\partial_{t}^{2 k-2-j} u(\xi, 0)\right|^{2}=-2 \operatorname{Re}\left(\partial_{t}^{2 k-1-j} u, \partial_{t}^{2 k-2-j} u\right) .
$$

Отсюда

$$
\begin{aligned}
& \left|\left(1+|\xi|^{2}\right)^{m} \sum_{j=0}^{k-2}(-1)^{k-j} \operatorname{Re} \partial_{t}^{2 k-2-j} u(0) \cdot \overline{\partial_{t}^{j} u(0)}\right| \leqslant \\
& \quad \leqslant \sum_{j=0}^{k-2}\left(2 \varepsilon_{1}\left(1+|\xi|^{2}\right)^{q j+q / 2}\left|\operatorname{Re}\left(\partial_{t}^{2 k-1-j} u, \partial_{t}^{2 k-2-j} u\right)\right|+\frac{1}{\varepsilon_{1}}\left(1+|\xi|^{2}\right)^{2 m-q j-q / 2}\left|\partial_{t}^{j} u(\xi, 0)\right|^{2}\right) .
\end{aligned}
$$


Применив для оценки первого слагаемого в правой части этого неравенства неравенство Коши, получим оценку

$$
\begin{aligned}
\left|\left(1+|\xi|^{2}\right)^{m} \sum_{j=0}^{k-2}(-1)^{k-j} \operatorname{Re} \partial_{t}^{2 k-2-j} u(0) \cdot \overline{\partial_{t}^{j} u(0)}\right| & \leqslant \\
\leqslant \sum_{j=0}^{k-2}\left(2 \varepsilon_{1}\left(1+|\xi|^{2}\right)^{q j+q / 2}\left(\varepsilon_{2}\left\|\partial_{t}^{2 k-1-j} u\right\|^{2}+\frac{1}{\varepsilon_{2}}\left\|\partial_{t}^{2 k-2-j} u\right\|^{2}\right)+\right. & \left.+\frac{1}{\varepsilon_{1}}\left(1+|\xi|^{2}\right)^{2 m-q j-q / 2}\left|\partial_{t}^{j} u(\xi, 0)\right|^{2}\right) .
\end{aligned}
$$

Выберем здесь $\varepsilon_{2}=\sqrt{\varepsilon_{1}}\left(1+|\xi|^{2}\right)^{-q / 2}$; тогда

$$
\begin{aligned}
& \mid\left(1+|\xi|^{2}\right)^{m} \sum_{j=0}^{k-2}(-1)^{k-j} \operatorname{Re} \partial_{t}^{2 k-2-j} u(0) \cdot \overline{\partial_{t}^{j} u(0)} \mid \leqslant \sum_{j=0}^{k-2}\left(2 \varepsilon_{1}^{3 / 2}\left(1+|\xi|^{2}\right)^{q j}\left\|\partial_{t}^{2 k-1-j} u\right\|^{2}+\right. \\
&\left.+2 \sqrt{\varepsilon_{1}}\left(1+|\xi|^{2}\right)^{q j+q}\left\|\partial_{t}^{2 k-2-j} u\right\|^{2}+\frac{1}{\varepsilon_{1}}\left(1+|\xi|^{2}\right)^{2 m-q j-q / 2}\left|\partial_{t}^{j} u(\xi, 0)\right|^{2}\right) .
\end{aligned}
$$

Применив это неравенство и неравенство Эрлинга-Ниренберга в правой части (28), получим

$$
\begin{aligned}
\|u\|_{2 m, \alpha, \frac{2 m}{2 k-1},|\xi|}^{2} \leqslant & c\left(\|A u\|^{2}+\varepsilon_{2}\left(\left\|\partial_{t}^{2 k-1} u\right\|^{2}+\left(1+|\xi|^{2}\right)^{2 m}\|u\|^{2}\right)+\right. \\
& \left.+c\left(\varepsilon_{2}\right) \sum_{j=0}^{k-2}\left(1+|\xi|^{2}\right)^{2 m-\frac{2 m}{2 k-1} j-\frac{m}{2 k-1}}\left|\partial_{t}^{j} u(\xi, 0)\right|^{2}+\left(1+|\xi|^{2}\right)^{m}\left|\partial_{t}^{k-1} u(\xi, 0)\right|^{2}\right) .
\end{aligned}
$$

Выбирая $\varepsilon_{2}>0$ достаточно малым, имеем

$$
\|u\|_{2 m, \alpha, \frac{2 m}{2 k-1},|\xi|}^{2} \leqslant c_{1}\left(\|A u\|^{2}+\sum_{j=0}^{k-1}\left(1+|\xi|^{2}\right)^{2 m-\frac{2 m}{2 k-1} j-\frac{m}{2 k-1}}\left|\partial_{t}^{j} u(\xi, 0)\right|^{2}\right) .
$$

Заметим теперь, что в силу условия 3

$$
\left|\partial_{t}^{j-1} u(\xi, 0)\right|=\left|\frac{\left.\sum_{|\tau| \leqslant m_{j}} b_{\tau j} \xi^{\tau} \partial_{t}^{j-1} u\right|_{t=0}}{\sum_{|\tau| \leqslant m_{j}} b_{\tau j} \xi^{\tau}}\right| \leqslant c(1+|\xi|)^{-m_{j}}\left|B_{j}(\xi) u\right|_{t=0}|\leqslant c| g_{j}(\xi) \mid .
$$

Применяя это неравенство в (29), получим оценку

$$
\|u\|_{2 m, \alpha, \frac{2 m}{3},|\xi|}^{2} \leqslant c\left(\|f\|^{2}+\sum_{j=1}^{k-1}\left(1+|\xi|^{2}\right)^{2 m-m_{j}-\frac{2 m}{2 k-1} j-\frac{m}{2 k-1}}\left|g_{j}(\xi)\right|^{2}\right) .
$$

Таким образом, доказана оценка (7) при $s=2 m$. Справедливость оценки (7) при $s>2 m$ доказывается методами, аналогичными методам работы [1].

\section{СПИСОК ЛИТЕРАТУРЫ}

1. Баев А. Д. Вырождающиеся эллиптические уравнения высокого порядка и связанные с ними псевдодифференциальные операторы// Докл. АН СССР. - 1982. - 265, № 5. - С. 1044-1046.

2. Баев А. Д. Качественные методы теории краевых задач для вырождающихся эллиптических уравнений. - Воронеж: Воронеж. гос. ун-т, 2008.

3. Баев А. Д. Об общих краевых задачах в полупространстве для вырождающихся эллиптических уравнений высокого порядка// Докл. РАН. - 2008. - 422, № 6. - С. 727-728. 
4. Баев А. Д., Бунеев С. С. Об одном классе краевых задач в полосе для вырождающихся эллиптических уравнений высокого порядка// Докл. РАН. - 2013. - 448, № 1. - С. 7-8.

5. Баев A. Д., Бунеев C. С. Априорная оценка решений одной краевой задачи в полосе для вырождающегося эллиптического уравнения высокого порядка// Вестн. Воронеж. гос. ун-та. Сер. Физ. Мат. 2012. - № 1. - C. 81-92.

6. Баев А. Д., Панков В. В. О существовании решений одного класса краевых задач в полосе для вырождающихся эллиптических уравнений высокого порядка// Докл. РАН. - 2017. - 475, № 5. - С. 1-3.

7. Вишик М. И., Грушин В. В. Краевые задачи для эллиптических уравнений, вырождающихся на границе области// Мат. сб. - 1969. - 80 (112), № 4. - С. 455-491.

8. Вишик М. И., Грушин В. В. Вырождающиеся эллиптические дифференциальные и псевдодифференциальные операторы// Усп. мат. наук. - 1970. - 25, № 4. - С. 29-56.

9. Глушко В. П. Априорные оценки решений краевых задач для одного класса вырождающихся эллиптических уравнений высокого порядка/ Деп. в ВИНИТИ 27.03.1979, № 1048. - Воронеж: ВГУ, 1979.

10. Панков B. В., Баев А. Д., Харченко В. Д. Об априорной оценке решений краевой задачи в полосе для вырождающегося эллиптического уравнения высокого порядка// Вестн. Воронеж. гос. ун-та. Сер. Физ. Мат. - 2018. - № 4. - С. 162-173.

Панков Владимир Владимирович

Воронежский государственный университет

E-mail: pankovfam@mail.ru

Баев Александр Дмитриевич

Воронежский государственный университет

E-mail: alexsandrbaev@mail.ru

Харченко Виктория Дмитриевна

Воронежский государственный университет

E-mail: dmitrieva9696@gmail.com

Бабайцев Андрей Александрович

Воронежский государственный университет

E-mail: 259608@mail.ru 